\title{
LA FORMACIÓN CONTINUA COLABORATIVA A TRAVÉS DE LA INVESTIGACIÓN-ACCIÓN. UNA FORMA DE CAMBIAR LAS PRÁCTICAS DE AULA
}

\author{
Raúl A. Barba-Martín \\ José J. Barba \\ Suyapa Martínez Scott \\ Universidad de Valladolid
}

RESUMEN: La formación continua del profesorado puede realizarse a través de diferentes modelos que sólo informan o que además acompañan en los procesos de poner en práctica las innovaciones. La formación, a través de procesos de reflexión, se plantea como fundamental para poder realizar transformaciones en las aulas, además si se hacen de manera colectiva con otros docentes o entre centros, las transformaciones no sólo se producen en el aula, sino que se dan en todo el contexto. Una de las formas de poder llevar una formación continua colaborativa es a través de grupos de investigaciónacción, considerándola como un conjunto de prácticas de carácter ético cuyas características permiten que los participantes se formen en función de sus necesidades y, a través del apoyo con otros docentes, con la finalidad de transformar el contexto. La investigación que presentamos está enmarcada en un PID de la Universidad de Valladolid, a través del cual docentes de tres centros educativos se han formado en educación inclusiva mediante investigación-acción implementando en sus aulas grupos interactivos. Este proceso de formación colaborativo, protagonizado por los propios docentes, ha producido transformaciones en su pensamiento, su aula y los contextos educativos en los que desarrollan su labor.

PALABRAS CLAVE: Formación continua, colaboración, investigaciónacción, innovación educativa, educación inclusiva.

\section{AN ONGOING COLLABORATIVE TEACHER TRAINING THROUGH ACTION RESEARCH. A WAY OF CHANGING CLASSROOM PRACTICES}

\footnotetext{
ABSTRACT: The ongoing education training for teachers can be done through different models that could only report or also accompany the process
} 
of implementing innovations. The training through reflection processes is presented as essential to make changes in the classroom; also, if it is done collectively with other teachers or between centers, transformations will not only occur in the classroom, but in the whole context. One way leading to a collaborative ongoing education is through action research groups, considering a set of ethical practices whose characteristics allow participants to be trained according to their needs, and through support with other teachers, in order to transform the context. The research we present here is framed in a Teaching Innovation Project, University of Valladolid, through which teachers from three schools that have been trained in inclusive education through action research implementing in their classrooms interactive groups.. This collaborative process played by teachers themselves has changed the thinking of teachers, their classroom and their educational contexts in which they work.

KEYWORDS: Ongoing learning, collaboration, action research, educational innovation, inclusive education.

Recibido: 30/04/2015

Aceptado: 30/07/2015

Correspondencia: Raúl A. Barba-Martín, Campus María Zambrano, Universidad de Valladolid, Plaza de la Universidad 1, 40004 Segovia. Email: raulbarba13@gmail.com.

\section{FORMACIÓN CONTINUA COLABORATIVA}

Los modelos tradicionales de formación, basados en el rol de expertos y aprendices, dificultan a los y las docentes la incorporación en sus aulas de los conocimientos adquiridos (Bevins y Price, 2014; López Pastor, Monjas y Manrique, 2011). Estos modelos se basan en el verbalismo o en el discurso teórico que según Freire (1968/2007) separan lo que se dice de lo que se hace, provocando que no se actúe en la realidad. El docente actúa como oyente pasivo y es el formador el encargado de seleccionar los contenidos, los objetivos, las estrategias metodológicas formativas y las actividades con las que supone que ayudará a los y las docentes a conseguir cambiar sus prácticas (González Calvo, 2013; Imbernón Muñoz, 2007). La dualidad de roles sin interacción hace que la formación no consiga vincular de una forma eficaz práctica y teoría, lo que dificulta que en las aulas se produzcan cambios durante un tiempo prolongado, siendo habitual que los conocimientos adquiridos queden limitados a momentos puntuales.

La formación que pretende transformaciones reales asentadas en el tiempo necesita que el docente cambie su pensamiento, su lenguaje y su forma de actuar, siendo un factor clave la reflexión. Havelock y Huberman (1977) afirman que nos podemos encontrar ante el "cambio sin el cambio", en el que los docentes realizan modificaciones para que todo siga igual. Por ejemplo, introducen un nuevo contenido pero siguen usando las mismas estrategias metodológicas, con lo que la realidad del aula sigue siendo la misma, ya que no ha habido un cambio real en los factores que 
pueden transformar la enseñanza. Así, siguiendo a Havelock y Huberman la verdadera transformación de la escuela ha de provenir de experiencias que transformen el contexto real. Para ello, el docente tiene que modificar sus concepciones educativas y, como consecuencia, sus metodologías y sus prácticas. Diferentes autores (Barba, González Calvo y Barba-Martín, 2014a; Elliot, 1986; González Calvo y Barba, 2014; Kincheloe, 2012; Schön, 1998; Stenhouse, 1987; Korthagen, 2007; Korthagen y Kessels, 2009; Perrenoud, 2010; Zeichner, 1993; Zeichner y Liston, 1996) consideran que este cambio de pensamiento docente se ha de hacer a través de procesos reflexivos del profesorado sobre su propia acción, ya sean éstos individuales o colectivos.

La formación basada en procesos individuales del profesorado permite modificar las prácticas de aula, pero no siempre un cambio en el pensamiento docente implica que se transforme realmente la educación que se imparte en el aula. La reflexión crítica es una de las formas más potentes de aprendizaje en maestros y maestras (Schön, 1998), a través de ella el profesorado abandona el peso de la tradición, y de que las cosas son como son, para comenzar a actuar como intelectuales críticos y transformadores dentro de su propia práctica (Giroux, 1990). Pero debemos tomar conciencia de que no toda la reflexión docente permite un cambio crítico del pensamiento. Hay una reflexión que viene a responder a las presiones de aula (Havelock y Huberman, 1977) que son decisiones que se han de tomar de forma rápida y precisa debido a la velocidad, inmediatez y complejidad con la que trascurren las cosas que suceden en el aula. Estas decisiones son reflexionadas pero no suelen provocar cambios profundos en el pensamiento docente, ya que se cuestiona los cómos y no los por qués, hay que tener en cuenta que la propia presión de aula no permite entrar a valorar cuestiones más allá de la inmediatez de la toma de decisiones. A esta forma de reflexión Zeichner y Liston (1996) la denominan reflexión-en-la-acción.

La reflexión profunda que transforma el pensamiento docente es la que se da alejada del aula, la que para Zeichner y Liston (1996) es la reflexión-sobre-la-acción. Este tipo de reflexión permite ahondar en profundidad en lo acontecido en el aula y vincularlo con sus conocimientos e inquietudes, pudiendo así encontrar los puntos débiles de las prácticas y mejorarlas en su totalidad (Barba-Martín, Barba y GómezMayo, 2014). Si bien, consideramos que estos procesos reflexivos han de ser organizados y sistemáticos teniendo en cuenta diferentes posibilidades: (a) el uso de diarios de los docentes, siendo escritos frecuentes en los que el o la docente reflexiona sobre su docencia y muestran su evolución en prácticas, metodología y pensamiento (Barba, González Calvo y Barba-Martín, 2014a; Moon, 2004, 2006; Porlan Ariza y Martín Toscano, 1991; Zabalza Beraza, 2004); (b) el uso de autobiografías y autoetnografías para tomar conciencia de la propia identidad, permitiendo tomar conciencia de lo que se ha hecho desde la perspectiva del presente y así transformar el propio pensamiento (González Calvo y Barba, 2014; Rivas, Leite y Cortes, 2014); y (c) los procesos reflexivos grupales, principalmente la investigación-acción como una forma grupal que permite analizar lo que sucede en el aula, debatirlo de forma colectiva y realizar nuevas acciones que lleven a su transformación (Barba y González Calvo, 2013; Kemmis y McTaggart, 1988; McKernan, 1996; Stringer, 2014; Zeichner, 2010).

El paso de la reflexión individual a la reflexión colectiva a través de la investigación-acción permite que las transformaciones no sólo se den en un aula, sino en todo el contexto. Kemmis y McTaggart (1988) afirman que si los cambios son colectivos, 
éstos son más potentes, transformando el discurso colectivo, las prácticas sociales y la organización social. Estas transformaciones grupales son clave cuando se aborda la transformación de un centro educativo, ya que son las que permitirán que el proyecto educativo sea un instrumento compartido que realmente represente la identidad del centro y las prácticas que allí se realizan, a partir de un proceso de construcción realmente democrático y participativo. La construcción de las prácticas y de la identidad colectiva a través de la investigación-acción, permite romper el aislamiento de la innovación en el aula y promover procesos colaborativos de reflexión, la solidaridad entre compañeros, el intercambio, el diálogo reflexivo y la planificación conjunta de experiencias innovadoras (Barba y González Calvo, 2014; Perrenoud, 2010).

Pero la investigación-acción también se puede desarrollar entre diferentes centros educativos provocando procesos de aprendizaje compartido. Hargreaves y Shirley (2012) definen la transferencia lateral como los aprendizajes de las mejores prácticas que se producen entre colegios cercanos. La investigación-acción, cuando se realiza entre docentes de diferentes centros educativos, permite que exista un espacio donde compartir experiencias, dificultades y buscar soluciones. Además, estos procesos realizados entre diferentes centros, ayudan a ampliar el conocimiento a través de buscar soluciones a una misma problemática compartida, lo que supone la generación simultánea de buenas prácticas. Entendiendo por buenas prácticas educativas las que reducen el abandono, el fracaso y la exclusión educativa, incrementando la cohesión social (Amores y Ritacco, 2012).

\section{LA INVESTIGACIÓN-ACCIÓN EN LA FORMACIÓN DE DOCENTES}

Compartimos la visión de Greenwood (2000) respecto a la investigación-acción como una forma de relación a través de compromisos intelectuales y éticos. En sus propias palabras "La investigación-acción no es una disciplina, ni una facultad, ni un método. Es un grupo de prácticas multidisciplinares orientadas a una estructura de compromisos intelectuales y éticos" (Greenwood, 2000: 32). Desde esta perspectiva se entiende la investigación más allá de una metodología basada en espirales, sino que la importancia se da a las cuestiones éticas y a la participación como una democrática real. De ahí que los "dueños del problema" (Greenwood, 2000) tomen las decisiones en todos los momentos del proceso, tales como: el diseño de procesos; la recopilación de preguntas y resultados; la evaluación de resultados de forma individual y colectiva; y la puesta en práctica de nuevas propuestas de mejora (Greenwood y Levin, 2007; Stringer, 2014). Los dueños del problema llevan a cabo la investigación en sus aulas y los métodos utilizados deben ir conformes a sus necesidades docentes para desarrollar su compromiso de democratizar su aula (Beane y Apple, 2005). No obstante, dentro de las múltiples formas de entender la investigación-acción Greenwood y Levin (2007) consideran que ésta siempre ha de tener tres componentes, que son:(a) acción; (b) participación; e (c) investigación.

\section{Acción}

La investigación-acción tiene como meta transformar el contexto donde se desarrolla a partir de la participación activa de los dueños del problema (Carr y Kemmis, 
1988; Contreras Domingo, 1994; Elliot, 1993; Kemmis y McTaggart, 1988). Por esta razón, el éxito o el fracaso se valorará en función del grado de cambio producido por las acciones diseñadas (Greenwood, 2000). Los y las docentes desarrollan sus propias propuestas en las aulas con el fin de mejorar los aprendizajes del alumnado y el clima del aula (Contreras Domingo, 1994; McKernan, 1996; Stenhouse, 1984). Las acciones desarrolladas no se quedan en lo anecdótico o en lo puntual, sino que persiguen mejoras duraderas a lo largo del tiempo que supongan verdaderas transformaciones estables.

El proceso de reflexión conjunta es fundamental para poder conseguir transformaciones sociales estables (Elliot, 1993). A través de la reflexión colectiva de las dificultades, de los problemas y de los logros, los docentes se plantean cómo transformar la acción. Así, hay que entender la acción como una parte de la praxis (Freire, 1968/2007), es decir, como un proceso que vincula la reflexión con la acción, no siendo adecuado darle un sentido de activismo, de actuar sin una reflexión sólida que apoye lo que se hace. A través de esta unión de reflexión con acción se van construyendo mejoras progresivas a partir de modificaciones o bien nuevas propuestas que dan respuesta a los problemas desde la perspectiva de sus dueños. La investigaciónacción busca generar una emancipación en los dueños del problema para que se conviertan en investigadores de su propia práctica y sean capaces de generar su propio conocimiento sin el apoyo del académico (Greenwood y Levin, 2007; Stringer, 2014). Pese a que la investigación-acción se ha de realizar entre los docentes y los académicos, no debemos de caer en modelos de investigación-acción más próximos a modelos basados en consultorías o en establecer grupos de investigación dependientes del académico, ya que pueden propiciar la imposición de visiones externas al grupo limitando la participación real (Barba, González Calvo y Barba-Martín, 2014b). Nuestra forma de entender la investigación supone un proceso de colaboración en un proyecto compartido que tiene como finalidad la emancipación de los dueños del problema. Así, los participantes aprenden a desenvolverse en la acción, generando soluciones a sus propios problemas a través de la reflexión colectiva. Al trabajar de manera continua sobre la propia acción los docentes se hacen expertos en la práctica y en la reflexión-sobre-la-práctica.

\section{Participación}

La investigación-acción establece una colaboración entre académicos y docentes, sin que haya un experto, o grupo de expertos, que tenga el control del proceso. Davydd Greenwood (2000) afirma que "la investigación-acción es una forma de investigación "co-generativa», en el sentido de que un grupo formado por los dueños legítimos del problema y un investigador profesional se reúnen para co-desarrollar sus metas investigadoras y para el cambio social" (p. 33). El proceso en el que está implicado todo el grupo es colaborativo, siendo el rol del investigador interno al grupo y no teniendo la potestad de dirigirlo (Greenwood y Levin, 2007). Estos procesos de relación igualitaria basada en el diálogo constante favorecen el desarrollo de una colaboración efectiva entre académicos y docentes (Bevins y Price, 2014). Es de resaltar el objetivo común y compartido de transformar el contexto en función de sus propias demandas y necesidades. 
Los docentes son los dueños del problema y participan de todos los momentos del proceso, desde el establecimiento de los objetivos en función de sus necesidades, a la elaboración de los resultados, pasando por las dinámicas de funcionamiento del grupo y los temas que se abordan. La investigación-acción es una forma de investigación al servicio del docente (Barba, 2013; McKernan, 1996). La búsqueda de mejoras en el contexto se realiza desde las necesidades de los propios participantes quienes demandan necesidades formativas para resolver sus problemas. Hay que tener en cuenta que son ellos mismos los que buscan formas de mejorar sus prácticas y de evaluar los resultados obtenidos. En este sentido, es un proceso de formación vinculado a sus propios intereses, a la mejora de sus conocimientos y a la vinculación de los debates y aprendizajes teóricos con la práctica, considerando que la participación activa es un proceso formativo integral.

Los y las docentes participantes, a través de la reflexión conjunta, desarrollan su propio conocimiento compartido sobre qué es la educación, la democracia en educación, la participación educativa y las prácticas de aula. Cuando los debates que están presentes en este modelo de investigación se dan entre diferentes centros educativos, se promueve la transferencia lateral del conocimiento entre los propios participantes, pudiendo aprender de las mejores prácticas y de las mejores soluciones a problemas similares dadas por otros centros educativos. A través de la participación en debates y reflexiones conjuntas acerca de experiencias, los docentes construyen soluciones y formas de abordar situaciones que ellos consideran problemáticas a la vez que se transforma la concepción educativa de las escuelas de la ciudad o de la comarca.

\section{Investigación}

La reflexión-sobre-la-acción constante por parte de los participantes les lleva a mejorar su capacidad investigadora sobre su propia práctica. La reflexión docente sobre la propia acción es uno de los aspectos claves en el aprendizaje del profesorado (Schön, 1998), si además se realiza de manera sistemática y organizada se convierte en investigación sobre su propia práctica (Zeichner y Liston, 1996). A través de la reflexión sistemática el docente aprende a analizar su propia práctica, a no culpabilizarse por las dificultades y a buscar soluciones.

La forma de desarrollar la investigación-acción es beneficiosa para el grupo, ya que aprende de otros iguales y alcanza una autonomía de investigación sobre su propia práctica que le permitirá en el futuro asumir un liderazgo en su aula y en su contexto para ser el percusor de transformaciones (Hargreaves y Shirley, 2012). En este sentido, nos encontramos con que la investigación-acción tiene un carácter emancipador, en el que el rol del investigador no es perpetuar un grupo de investigación, sino que consiste en promover transformaciones sociales y dotar de autonomía a los dueños del problema para que puedan resolver nuevos problemas por sí mismos. Además de esta labor, el investigador académico también ha de buscar la transferencia entre contextos del proyecto, para que pueda ser aprovechado por otras instituciones educativas, así como explicar de forma clara el proyecto desarrollado (Greenwood, 2000). 


\section{LA VINCULACIÓN DE ESCUELAS Y LA UNIVERSIDAD A TRAVÉS DE UN PROYECTO DE INNOVACIÓN DOCENTE SOBRE EDUCACIÓN INCLUSIVA}

El marco de un Proyecto de Innovación Docente (PID) de la Universidad de Valladolid nos permitió comenzar a trabajar con docentes de tres centros educativos sobre educación inclusiva mediante investigación-acción. Al construir el proyecto contábamos con un pequeño grupo de profesores de universidad, cuatro docentes de infantil y primaria con los que compartíamos la forma de entender la educación inclusiva y la necesidad de actuar en las aulas. Entendemos que la educación inclusiva es un proceso de eliminación de barreras, especialmente de los colectivos y estudiantes más débiles, que permite al alumnado tener presencia, participar y aprender en el centro educativo en el que están escolarizados (Ainscow, Booth y Dyson, 2006). Si bien nuestras experiencias y concepciones partían de los principios y convicciones de la investigación-acción-participativa, también nos veíamos reforzados por la idea de Ainscow y colaboradores (Ainscow, Booth y Dyson, 2004; Ainscow, Howes, Farrell y Frankham, 2004) que consideran la investigación-acción como un método adecuado para transformar los colegios en escuelas inclusivas. La ilusión por comenzar el proyecto hizo que los cuatro docentes convencieran a compañeros y compañeras de su centro para participar, siendo diecisiete en la primera toma de decisiones.

La primera decisión partió de los y las docentes, ya que consideraban necesario adquirir una mejor formación sobre educación inclusiva y conocer experiencias concretas antes de comenzar a desarrollar prácticas de este tipo en sus aulas. Tras realizar un curso en el que, a través de presentar diferentes propuestas de centros educativos se revisaban los conceptos teóricos, llegó la hora de comenzar a plantearnos cómo actuar en las aulas. Así, a través de un proceso reflexivo conjunto de análisis de las diferentes realidades, se valoró cuáles eran los aspectos de la educación inclusiva que los y las docentes querían mejorar en sus contextos y, además, con qué tipo de prácticas podrían conseguir mejores resultados en este sentido.

Con respecto a la segunda decisión, los y las docentes valoraron de forma muy positiva poder vincular la mejora de la educación inclusiva con la mejora de la participación familiar, para lo que eligieron realizar grupos interactivos. (Elboj y Nimela, 2010; Valls y Kiriades, 2013). Esta decisión Ilevó aparejada una nueva demanda por parte de los y las docentes, que fue un nuevo proceso de formación teórica sobre grupos interactivos. Tras esta formación, en las reuniones se entremezclaba la ilusión por desarrollar la nueva práctica que permitiría mejorar la educación inclusiva y la participación familiar, junto con las dudas por las nuevas demandas docentes y por que las familias entraran al aula. El proceso formativo en grupos interactivos se construyó con los y las docentes como epicentro, ya que a partir de la narración de sus experiencias en las sesiones se establecía la colaboración con los demás para transformar las prácticas educativas en sus aulas y, por ende, en las escuelas en las que trabajaban. Por parte de las escuelas ha supuesto una valoración positiva de esta forma de trabajo frente a metodologías más tradicionales con las que acostumbraban a formarse.

Un aspecto clave en el proceso de innovación en las aulas era la reunión mensual en la que participábamos los miembros del proyecto, exponiendo casos concretos de prácticas de aula, estableciendo similitudes y diferencias con otras y buscando 
soluciones a las dificultades surgidas. Las soluciones emergían del propio colectivo, generando conocimiento a partir de ello. La dinámica de funcionamiento se basaba en que todas las personas podían aportar lo que considerasen oportuno, si bien en el caso de los investigadores había un especial cuidado en la participación para no monopolizar el debate e imponer su criterio, siendo habitual que sus intervenciones se realizaran a través de preguntas a los y las docentes. El grupo había creado un espacio de debate acorde a sus necesidades en el que exponían datos y reflexiones que les habían surgido en las sesiones previas a la reunión para ser abordadas de forma colaborativa.

La recogida de datos se ha realizado de múltiples formas. Las reuniones han sido grabadas para después ser analizadas. Estos datos han sido triangulados con los que se obtenían a través de otras herramientas: (a) un cuestionario abierto que se pasó al empezar el Proyecto, para conocer las expectativas y necesidades de los participantes; (b) entrevistas, que se realizaron cuando acabó el primer año del Proyecto para conocer las aportaciones que este tipo de formación había tenido individualmente en cada docente; y (c) observaciones en las aulas, que se realizaban cuando los participantes del PID que no éramos maestros y maestras entrábamos como voluntariado cultural en los grupos interactivos. Para la redacción de este artículo hemos elaborado una narrativa del proceso (Greenwood y Levin, 2007).

\section{Gestión de la participación familiar}

La principal acción Ilevada a cabo durante el desarrollo del Proyecto fue la implementación de nuevas prácticas de educación inclusiva en las aulas. Los y las docentes decidieron poner en práctica en sus aulas grupos interactivos siendo éste un proceso de innovación docente abordado de una forma colectiva. Al participar de la investigación-acción se consiguió que la formación no quedase únicamente en la base teórica de qué son y cómo se hacen, sino que las y los docentes fueron los encargados de llevar a cabo la acción, de implementarlos, de debatir sobre las dificultades encontradas y logros conseguidos para contrastar la teoría. La labor semanal de los y las docentes preparando materiales para grupos interactivos, gestionando las actividades y el tiempo, formando al voluntariado previamente al trabajo con el alumnado, evaluándolo, etc., no se desarrollaba de manera individual, ya que se contaba con el apoyo del resto del grupo para no desfallecer y encontrarse en soledad.

La acción no se quedó solo en la transformación de las aulas, sino que los docentes transformaron la relación con las familias para que participaran como voluntarias en grupos interactivos. Incluir a los adultos de la comunidad en las aulas conllevaba la necesidad de una organización familiar novedosa y compleja. Los y las docentes de cada centro educativo se coordinaron para reunirse con las familias explicándoles en qué consistía la nueva actuación, lo necesaria que era su participación y las ventajas que conllevaba. La primera cuestión que se trató en las reuniones era la gestión de los voluntarios y voluntarias y cómo asegurarse que al empezar las sesiones hubiera suficiente voluntariado, etc. El colectivo decidió que lo más apropiado sería que en cada centro una persona vinculada al proyecto gestionara las cuestiones del voluntariado y su organización en las aulas a través de listas planificadas con días de antelación. El grado de complejidad fue diferente entre las aulas unitarias, donde la mayor 
dificultad se encontró en que muchos familiares repetían muy a menudo, y los colegios con siete aulas, que llegaron al centenar de voluntarios y voluntarias. Resueltos los primeros problemas de organización los y las docentes decidieron aprovechar la inclusión del voluntariado en las aulas para conseguir mejorar la vida del centro. Así, se plantearon que los familiares conociesen al alumnado de aulas diferentes a las de sus hijos e hijas. En el caso de los colegios grandes, con dos o tres líneas por curso, existía la preocupación de que las familias conocieran al resto de docentes implicados, a los niños y niñas del colegio y a familias con hijos e hijas en otras aulas, por esta razón, se creyó necesario que los familiares rotasen por las diferentes clases. Se consideró que esta era una manera de comenzar a crear una gran comunidad en el centro y que las familias traspasaran las fronteras del aula.

Ante la baja participación de familias inmigrantes en grupos interactivos se planteó un debate en el grupo para promoverlo. Nos encontramos con que la dificultad con el idioma hacía que participasen poco en la vida del centro. Si bien se cumplía un primer objetivo que es que las familias del lugar conocieran y se relacionaran de una forma positiva con niños y niñas inmigrantes, faltaba la segunda parte del proceso inclusivo, que las familias inmigrantes participaran de la escuela. Tras debatir diferentes soluciones, el grupo decidió que los y las docentes buscaran momentos para dialogar y convencerlos de que al menos probasen a ir una vez. Esto acabó surtiendo efecto y algunos familiares inmigrantes se animaron a participar viviendo una experiencia positiva para ellos, para sus hijos e hijas y para el resto de la comunidad. Esto provocó un efecto de contagio animando a otras familias inmigrantes a participar.

\section{Actuaciones en el aula}

Una vez que los y las docentes conocieron diferentes actuaciones inclusivas para llevar a cabo en el aula, definieron el objetivo que querían conseguir a través de la formación. Los profesores universitarios decidieron que para que el proceso fuese realmente participativo, eran los y las docentes quienes debían elegir qué actuación desarrollar en función de sus intereses y de sus aulas. Tras un análisis de las diferentes realidades, se llegó al acuerdo de que se quería fomentar la participación familiar y la inclusión a través de grupos interactivos. Las cuestiones debatidas en el colectivo, en cuanto a su puesta en práctica, se realizaron en torno a dos aspectos: (a) el diseño de actividades; y (b) el papel del voluntariado dentro del grupo.

Diseño de actividades, los y las docentes tenían preocupaciones respecto a realizar actividades que conllevaran interacciones entre los niños y niñas con los adultos. El trabajar de forma colaborativa con docentes de otros colegios permitió apoyarse de forma conjunta y debatir sobre qué aspectos modificar para hacer las prácticas más inclusivas y dialógicas, siendo éstas: (a) evitar poner actividades individuales para que tuviesen que compartirlo, debido a que habían comprobado que si no se centraban mucho en su tarea y se olvidaban de los compañeros y compañeras; y (b) exigir que hubiera diálogo y consenso entre el alumnado antes de comenzar las tareas. Esta aportación fue realizada por una maestra que lo había observado de un voluntario, lo expuso al grupo y se consideró una opción muy positiva ya que fomentaba el diálogo.

El papel del voluntario o voluntaria dentro del grupo. A los docentes les daba respeto corregir al voluntariado que, en grupos interactivos, en lugar de generar inte- 
racciones entre el alumnado guiaba sus actividades. Estas nuevas relaciones planteaban muchas dudas, ya que no sabían cómo reorientar la participación del familiar, si entrando disimuladamente en el grupo y dando pequeñas pautas, si diciéndoselo directamente a él, o bien hablando fuera de los grupos explicándole un poco los errores vistos. Tras varios debates colectivos entre los miembros del grupo decidieron que la mejor manera era hacer un recordatorio antes de comenzar los grupos interactivos respecto al papel del voluntariado si bien esta actuación no fue del todo eficaz. A partir de la experiencia de docentes que habían probado a entrar en el grupo interactivo a dar alguna pauta al voluntariado, se debatió esta forma de actuar y se consideró que era la opción más adecuada, avalada por que en muchos casos las personas que actuaban de voluntarias habían agradecido la corrección.

\section{Evaluación y difusión de resultados}

A lo largo del texto hemos mostrado cómo generando un espacio de diálogo mediado por el profesorado universitario, los propios docentes no necesitaban de un experto que evaluase y diera solución a sus dificultades. Los mismos docentes eran quienes llevaban todo el peso de la evaluación de su actuación y, a partir de un proceso de reflexión-sobre-la-acción, se estaba constituyendo un proceso de evaluación formativa y colectiva que les permitió encontrar las soluciones a las demandas que ellos mismos descubrían.

Estos procesos sistemáticos de evaluación y de búsqueda de la mejora de la práctica docente han convertido a los docentes de las escuelas en investigadores e investigadoras de su propia práctica. Así, han participado en congresos y en jornadas contando las experiencias que han desarrollado en sus aulas haciendo especial énfasis en los procesos personales y escolares de innovación educativa. Este carácter investigador, adquirido por el proceso, les confiere una emancipación que les permite afrontar la innovación educativa y la solución de futuros problemas de forma autónoma, siendo esta característica compartida por el grupo.

\section{Discusión}

La reflexión docente es una de las herramientas más favorecedoras para desarroIlar la formación continua del profesorado debido a que tiene sentido desde su propia realidad educativa, si bien afrontar esto desde una dimensión colectiva permite lograr mayores metas. En el análisis del PID que ha sido expuesto, los y las docentes han transformado sus prácticas educativas en función de la reflexión sobre sus necesidades y de la búsqueda de soluciones colectiva. El conocimiento profundo de sus propias realidades les ha ayudado a definir los problemas con los que no estaban conformes, a observar y reflexionar para analizarlos, y a buscar soluciones individuales y colectivas para cambiarlos. A través de la investigación-acción se consigue que la formación docente parta de las necesidades de los y las participantes (Liston y Zeichner, 2003; Woods, 1987). Los procesos reflexivos colaborativos guiaron al PID hacia el fomento de la participación familiar en las aulas. Durante varios meses los y las docentes estuvieron realizando grupos interactivos y reflexionando constantemente sobre su propia práctica para dar solución a los problemas que les surgieron, 
tales como: ¿qué tipo de actividades hacer? ¿qué papel adoptar durante los grupos interactivos? ¿cómo fomentar la participación de familias inmigrantes? ¿cómo tratar con el voluntariado? Estos problemas se solucionaron con la práctica continua y a través de las experiencias de los compañeros y compañeras y, actualmente, son estos docentes los que tranquilizan a los nuevos participantes del grupo que tienen las mismas dudas que ellos cuando empezaron.

La realización del PID más allá del ámbito de un único centro educativo ha permitido transformaciones más allá del aula y del centro. Se ha comenzado a plantear la educación inclusiva como una realidad que va mas allá de un aula o de un centro educativo generando lenguajes y experiencias que sirven como forma de diálogo con compañeros y compañeras de otros centros. Al final se han desarrollados unas prácticas y un lenguaje propio del grupo independientemente del aula, del docente o del tipo de centro educativo. Además, la unión de profesores y profesoras más allá de las fronteras de un centro, pero con intención de innovar en sus prácticas de aula sobre un aspecto común -la educación inclusiva- ha permitido que se perdiera el miedo al cambio. La aportación continua de los participantes para solucionar problemas reales descubiertos en sus contextos por ellos mismos, ayudó a que se realizasen propuestas sólidas y a que rápidamente se animaran a buscar nuevos retos para no dejar de mejorar. Nuestra investigación ratifica lo enunciado por Ainscow y colaboradores (Ainscow et al., 2004; Ainscow, Howes, Farrell y Frankham, 2004; Howes, Frankham, Ainscow y Farrell, 2004) referente a que una de las mejores formas de vincular la investigación escolar sobre inclusión y los equipos multinivel es la investigaciónacción. EI PID ha dado respuesta a las demandas de todos los y las participantes, pese a su perfil variado, ya que había profesorado universitario, con maestros y maestras en ejercicio y con estudiantes de Grado de Maestro y del Máster en Investigación. Al profesorado de universidad le ha permitido co-aprender con docentes que buscaban mejorar sus prácticas educativas. Han podido acceder de primera mano a las necesidades y problemas que maestros y maestras tienen en los centros escolares y a conocer cómo solucionarlos, uniendo práctica y teoría (Freire, 1968/2007). A los y las estudiantes universitarios les ha permitido conocer la realidad educativa, a veces tan alejada de la formación inicial (Barba, 2006), y poder así desarrollar sus Trabajos de Fin de Grado y Trabajos Fin de Máster sobre educación inclusiva a través de una experiencia vivida en contextos escolares.

La formación no consistió en aprender una técnica y ponerla en práctica, sino que en función de las dificultades y de los replanteamientos se buscaron las formas más eficaces para desarrollarlo. No se conformaron con saber qué eran grupos interactivos y ponerlo en práctica, sino que se buscó cómo sacar el máximo rendimiento en cada aula. Gracias a esto, se han llegado a producir grandes cambios en las aulas como el de un docente que antes de participar en el Proyecto no realizaba dinámicas de pequeño grupo en su aula -como el trabajo por rincones- y al finalizar había superado el miedo a perder el control del aula. Ahora este docente está muy comprometido con las prácticas grupales y el diálogo en su aula, lo que antes consideraba complicadísimo, ahora lo considera sencillo y favorecedor de los aprendizajes. Este cambio ha sido posible gracias a que la formación del profesorado se ha desarrollado mediante la reflexión docente (Schön, 1998). El docente ha transformado su pensamiento a través de procesos reflexivos en los que analizaba su propia práctica (Kin- 
cheloe, 2012; Stenhouse, 1987; Perrenoud, 2010). Este cambio en sus concepciones educativas ha permitido las transformaciones en su aula que se han asentado en el tiempo (Havelock y Huberman, 1977; Schön, 1998; Zeichner, 1993).

La investigación-acción, ha sido la forma de unir los procesos de reflexión-sobre la-acción y el carácter colectivo de la formación continua para facilitar a los y las docentes la puesta en práctica de propuestas de mejora en sus aulas, a la vez que se vinculaban con la Universidad. El rol de los universitarios ha sido de acompañante del proceso ayudando en las demandas de los y las docentes, a la reflexión y al análisis de las actuaciones. El profesorado universitario fue el encargado de especificar, junto con el resto de miembros, los objetivos del Proyecto al principio y de cuidar que se tuviesen en cuenta todas las variables posibles. Esto favoreció que el PID haya funcionado a través de la colaboración efectiva entre académicos y docentes, alejándose de relaciones del tipo cliente-proveedor que hubiesen hecho fracasar la cooperación prevista entre los miembros (Bevins y Price, 2014).

Hay que tener en cuenta que el otro logro de los académicos ha sido generar autonomía en estas situaciones para el grupo de docentes, que son capaces de realizar grupos interactivos y colaborar con sus compañeros y compañeras. Los y las docentes son capaces de innovar de forma autónoma con grupos interactivos, lo que supone que la investigación-acción ha generado un proceso emancipatorio (Contreras Domingo, 1994; Greenwood y Lewin, 2007; Stringer, 2014). Por ello, el académico deja de ser una figura necesaria en el proceso en el que ya se han formado y su papel pasa a ser el de iniciador de nuevos retos. En el caso del PID, los participantes, al finalizar el primer año, admitían tener autonomía para realizar grupos interactivos pero no para desarrollar nuevas propuestas inclusivas en las aulas. Por esta razón, al evaluar el PID un grupo numeroso de docentes consideraron que en el siguiente curso académico el grupo debía de seguir trabajando en nuevas líneas como las tertulias literarias dialógicas.

El futuro del Proyecto tiene como horizonte la realización de nuevas prácticas inclusivas y afianzar las ya trabajadas en las aulas y en los nuevos miembros del grupo. La intención es seguir dando cabida a todas las necesidades, grupales e individuales, tanto de los miembros que han estado el primer año, como de los que entran nuevos. Además, nos enfrentamos al reto del paso de los docentes como investigadores de sus propias prácticas, aspecto que se está empezando a trabajar a través de la realización de Trabajos de Fin de Grado y de Trabajo Fin de Máster de los docentes sobre los procesos de innovación de sus aulas. Pero no hemos de perder el referente de que el PID tiene un eje común, y con una única finalidad, que las acciones realizadas produzcan cambios positivos en las aulas, en las escuelas, y en las comunidades, fomentando una mayor justicia social y una concepción más ética de la educación.

\section{REFERENCIAS BIBLIOGRÁFICAS}

Ainscow, M., Booth, T., y Dyson, A. (2004). Understanding and developing inclusive practices in schools: A collaborative action research network. International Journal of Inclusive Education, 8(2), 125-139. doi: 10.1080/1360311032000158015. 
Ainscow, M., Booth, T., y Dyson, A. (2006). Improving schools, developing inclusion. Londres: Routledge.

Ainscow, M., Howes, A., Farrell, P., y Frankham, J. (2004). Investigación-acción: Una propuesta para el desarrollo de prácticas inclusivas. Cuadernos de Pedagogía, 331, 54-59.

Amores, F.J., y Ritacco, M. (2012). Prácticas escolares ante la exclusión social. Estudio en la Educación secundaria obligatoria. Contextos Educativos: Revista de Educación, 15, 41-60. doi: 10.18172/con.654.

Barba, J.J. (2006). Aprendiendo a ser maestro en una escuela unitaria. Vivencias, sensaciones y reflexiones en la primera oportunidad. Morón (Sevilla): MCEP.

Barba, J.J. (2013). La investigación cualitativa en educación en los comienzos del siglo XXI. En M. Díaz y A. Giráldez (Coords.). La investigación cualitativa en educación musical (pp. 23-38). Barcelona: Graó.

Barba, J.J., y González Calvo, G. (2013). Perseguir el sueño o caer en lo que existe: La socialización del maestro novel entre el habitus y la investigación-acción. Revista Interuniversitaria de Formación del Profesorado, 78(27, 3), 137-144.

Barba, J.J., González Calvo, G., y Barba-Martín, R.A. (2014a). El uso de los diarios del profesorado como instrumento de reflexión-sobre-la-acción. Revista Española de Educación Física y Deportes, 405, 55-63.

Barba, J.J., González Calvo, G., y Barba-Martín, R.A. (2014b). Qué la fuerza esté contigo: Desvelar el lado oscuro de la investigación en educación. Magis: Revista Internacional de Investigación en Educación, 7(14), 125-140. Recuperado de http://revistas.javeriana.edu.co/index.php/MAGIS/article/view/11861/pdf.

Barba-Martín, R., Barba, J.J., y Gómez-Mayo, P. (2014). El papel crítico y reflexivo del profesorado ante el aprendizaje cooperativo. Revista Digital EmásF, 29, 8-18. Recuperado de: http://emasf.webcindario.com/El_papel_critico _y_reflesivo_del_ profesorado_ante_el_aprendizaje_cooperativo.pdf.

Beane, J.A., y Apple, M.W. (2005). La defensa de las escuelas democráticas. En M.W. Apple y J.A. Beane (Coords.), Escuelas democráticas (pp. 13-47). Madrid: Morata.

Bevins, S., y Price, G. (2014). Collaboration between academics and teachers: a complex relationship. Educational Action Research, 22(2), 270-284. doi: 10.1080/09650792.2013.869181.

Carr, W., y Kemmis, S. (1988). Teoría crítica de la enseñanza. Barcelona: Martínez Roca.

Contreras Domingo, J. (1994). La investigación en la acción: ¿Qué es? Cuadernos de Pedagogía, 224, 8-12.

Elboj, C., y Niemela, R. (2010). Sub-communities of mutuals learners in the classroom. The case of interactive groups. Revista de Psicodidáctica, 15(2), 177-189. doi: 10.1387/RevPsicodidact.810.

Elliot, J. (1986). La investigación-acción en el aula. Valencia: Generalitat Valenciana.

Elliot, J. (1993). El cambio educativo desde la investigación-acción. Madrid: Morata.

Freire, P. (2007). Pedagogía del oprimido (16 a ed.). Madrid: Siglo XXI.

Giroux, H.A. (1990). Los profesores como intelectuales. Hacia una pedagogía crítica del aprendizaje. Barcelona: Paidós/MEC. 
González Calvo, G. (2013). Evolución de la identidad profesional de un docente novel de Educación Física: Análisis a partir de la reflexión de sus experiencias personales y de su propia práctica. Tesis Doctoral. Valladolid: Universidad de Valladolid.

González Calvo, G., y Barba, J.J. (2014). Formación permanente y desarrollo de la identidad reflexiva del profesorado desde las perspectivas grupal e individual. Profesorado. Revista de Currículum y Formación del Profesorado, 18(3), 397-412. Recuperado de http://www.ugr.es/local/recfpro/rev181COL12.pdf.

Greenwood, D.J. (2000). De la observación a la investigación-acción participativa: una visión crítica de las prácticas antropológicas. Revista de Antropología Social, 9, 27-49. Recuperado de http://revistas.ucm.es/index.php/RASO/article/view/RASO0000110027A/9940.

Greenwood, D. J., y Levin, M. (2007). Introduction to action research. social research for social change ( $2^{\mathrm{a}} \mathrm{ed}$.). Thousand Oaks, CA: Sage.

Havelock, R.G., y Huberman, A.M. (1977). Solving educational problems. The theory and reality of innovation in developing countries. Geneve: UNESCO-OIE. Recuperado de http://unesdoc.unesco.org/images/0002/000297/029794eo.pdf.

Hargreaves, A., y Shirley, D. (2012). La cuarta vía. El futuro prometedor del cambio educativo. Barcelona: Octaedro.

Howes, A., Frankham, J., Ainscow, M., y Farrell, P. (2004). The action in action research: Mediating and developing inclusive intentions. Educational Action Research, 12(2), 239-258. doi:10.1080/09650790400200247.

Imbernón Muñoz, F. (2007). Diez ideas clave: la formación permanente del profesorado. Nuevas ideas para formar en la innovación y el cambio. Barcelona: Graó.

Kemmis, S., y McTaggart, R. (1988). Como planificar la investigación-acción. Barcelona: Laertes.

Kincheloe, J. L. (2012). Teachers as researches. Qualitative inquiry as a path to empowerment $\left(4^{\mathrm{a}} \mathrm{ed}\right.$.). Wiltshire: Routledge.

Korthagen, F. (2007). The gap between research and practice revised. Educational Research and Evaluation, 13(3), 303-310. doi: 10.1080/13803610701640235.

Korthagen, F., y Kessels, J. (2009). Linking theory and practice: Changing the pedagogy of teacher education. Educational Research, 28(4), 4-17. doi: 10.3102/ $0013189 \times 028004004$.

Liston, D.P., y Zeichner, K.M. (2003). Formación del profesorado y condiciones sociales de la escolarización. Madrid: Morata.

López-Pastor, V.M., Monjas, R., y Manrique, J.C. (2011). Fifteen years of action research as professional development: Seeking more collaborative, useful and democratic systems for teachers. Educational Action Research, 19(2), 153-170. doi: 10.1080/09650792.2011.569190.

McKernan, J. (1996). Investigación-acción y currículum. Métodos y recursos para profesionales reflexivos. Madrid: Morata.

Moon, J.A. (2004). Reflection in learning \& professional development ( $2^{\mathrm{a}} \mathrm{ed}$.). Abingdon, Oxon: Routledge Falmer.

Moon, J.A. (2006). Learning journals. A handbook for reflective practice and professional development ( $3^{\mathrm{a}}$ ed.). New York, NY: Routledge Falmer. 
Perrenoud, P. (2010). Desarrollar la práctica reflexiva en el oficio de enseñar: profesionalización y razón pedagógica. Barcelona: Graó.

Porlán Ariza, R., y Martín Toscano, J. (1991). El diario del profesor. Un recurso para la investigación en el aula. Sevilla: Diada.

Rivas, J.I., Leite, A., y Cortés, P. (2014). Formación del profesorado y experiencia escolar: las historias de vida como práctica educativa. Praxis Educativa, 18(2), 13-23. Recuperado de http://ojs.fchst.unlpam.edu.ar/ojs/index.php/praxis/article/ view/909.

Schön, D.A. (1998). El profesional reflexivo. Cómo piensan los profesionales cuando actúan. Barcelona: Paidós.

Stenhouse, L. (1984). Investigación y desarrollo del currículum. Madrid: Morata.

Stenhouse, L. (1987). La investigación como base en la enseñanza (5 a ed.). Madrid: Morata.

Stringer, E.T. (2014). Action research (4 ${ }^{\mathrm{a}}$ ed.). Thousand Oaks, CA: SAGE.

Valls, R., y Kyriakides, L. (2013). The power of interactive groups: how diversity of adults volunteering in classroom groups can promote inclusion and success for children of vulnerable minority ethnic populations. Cambridge Journal of Education, 43(1), 17-33. doi: 10.1080/0305764X.2012.749213.

Woods, P. (1987). La escuela por dentro. Barcelona: Paidos.

Zabalza Beraza, M. A. (2004). Diarios de clase: Un instrumento de investigación y desarrollo profesional. Madrid: Narcea.

Zeichner, K.M. (1993). El docente como profesional reflexivo. Cuadernos de Pedagogía, 220, 44-49.

Zeichner, K.M. (2010). La formación del profesorado y la lucha por la justicia social. Madrid: Morata.

Zeichner, K.M., y Liston, D.P. (1996). Reflective teaching. An introduction. Mahwah, NJ: Lawrence Erlbaum Associates. 